\title{
Trial-to-Trial Variability and State-Dependent Modulation of Auditory-Evoked Responses in Cortex
}

\author{
Michael A. Kisley and George L. Gerstein \\ Department of Neuroscience, University of Pennsylvania School of Medicine, Philadelphia, Pennsylvania 19104-6074
}

Recent experimental work has provided evidence that trial-totrial variability of sensory-evoked responses in cortex can be explained as a linear superposition of random ongoing background activity and a stationary response. While studying single trial variability and state-dependent modulation of evoked responses in auditory cortex of ketamine/xylazine-anesthetized rats, we have observed an apparent violation of this model.

Local field potential and unit spike trains were recorded and analyzed during different anesthesia depths-deep, medium, and light-which were defined by the pattern of ongoing cortical activity. Estimation of single trial evoked response was achieved by considering whole waveforms, rather than just one or two peak values from each wave. Principal components analysis was used to quantitatively classify waveforms on the basis of their time courses (i.e., shapes).

We found that not only average response but also response variability is modulated by depth of anesthesia. Trial-to-trial variability is highest under medium levels of anesthesia, during which ongoing cortical activity exhibits rhythmic population bursting activity. By triggering the occurrence of stimuli from the spontaneously occurring burst events, we show that the observed variability can be accounted for by the background activity. In particular, the ongoing activity was found to modulate both amplitude and shape (including latency) of evoked local field potentials and evoked unit activity in a manner not predicted by linear superposition of background activity and a stereotyped evoked response. This breakdown of the linear model is likely attributable to rapid transitions between different levels of thalamocortical excitability (e.g., spike-wave discharges), although brain "state" is relatively fixed.

Key words: variability; modulation; local field potential; principal components analysis; anesthesia; ketamine; xylazine; auditory cortex; spike-wave discharge; high-voltage spindle
On a trial-by-trial basis, cortical activity evoked by sensory stimulation is extremely variable. This has been shown for both single units (Schiller et al., 1976; Whitsel et al., 1977; Heggelund and Albus, 1978; Rose, 1979; Tolhurst et al., 1983; Scobey and Gabor, 1989; Vogels et al., 1989; Softky and Koch, 1993) (but see Gur et al., 1997) and macropotentials, especially human evoked potentials (for review, see Childers et al., 1987) (also see Thomas et al., 1989; Liberati et al., 1991). The traditional method for dealing with such variability is averaging over many trials [units: Gerstein (1960); evoked potentials: Dawson (1951); for review, see Aunon et al. (1981)]. Such a manipulation requires the assumption of linear superposition between basically random ongoing background activity and a highly stereotyped, repeatable evoked response. Recently, voltage-sensitive dye (Arieli et al., 1996) and intracellular (Azouz and Gray, 1999) recordings from the visual cortex of anesthetized cats have provided support for the validity of this assumption. Nevertheless, nonstationarity of general brain state can lead to nonstationarity of the so-called "repeatable" response (for review, see Coenen, 1995) (also see Discussion), thus violating the assumptions necessary for averaging (Coppola et al., 1978; Möcks et al., 1987). In the present report we dem-

Received May 17, 1999; revised Aug. 30, 1999; accepted Sept. 13, 1999. This work was supported by Grants MH 46428 and DC 01249 from National Institutes of Health. We gratefully acknowledge Dr. Jeff Keating, Sanjiv Talwar, and Dr. Stuart Baker for technical assistance and helpful discussions, and Drs. Pawel Musial and Larry Palmer for comments on an earlier version of this manuscript.

Correspondence should be addressed to Michael A. Kisley, Department of Neuroscience, 215 Stemmler Hall/6074, University of Pennsylvania, Philadelphia, PA 19104-6074. E-mail: mike@mulab.physiol.upenn.edu.

Copyright (C) 1999 Society for Neuroscience 0270-6474/99/1910451-10\$05.00/0 onstrate a violation of linearity between ongoing and evoked activity in rat auditory cortex even at a stable plane of anesthesia.

Traditionally, trial-to-trial variability studies of auditory evoked potentials in subhuman species have involved characterizing each individual waveform with only one or two points per waveform (Tunturi, 1959; Worden et al., 1964; Horvath, 1969). Such simple measures of single trial evoked responses, such as the amplitude between two peaks, might not always be sufficiently sensitive to detect modifications of waveform time course. Principal components analysis (PCA), on the other hand, provides a method for quantifying the shape of an entire single trial waveform. This type of analysis has seen increasing application in neuroscience, including sorting of extracellularly recorded action potential waveforms (Abeles and Goldstein, 1977), analysis of evoked potentials (for review, see Chapman and McCrary, 1995), synaptic potentials (Astrelin et al., 1998), evoked extracellular field potentials (Musial et al., 1998), and current source density waveforms (Di et al., 1990). In the present study we use PCA to quantify waveform variability, and further to reduce variability by numerically classifying single trial evoked field potential responses into groups of similarly shaped waveforms.

By recording evoked local field potential and unit activity from the auditory cortex of rats anesthetized with ketamine/xylazine, we have endeavored to (1) quantify single trial evoked responses and their variability using whole waveforms rather than just one or two points, (2) examine the modulation of response and response variability by the different brain states available with our anesthetic regimen, (3) investigate the impact of ongoing activity (as the potential cause of variability), and (4) compare the variation in local field potential evoked responses with that of neu- 
rons. Our results are discussed in the context of thalamocortical rhythms and spike-wave discharges.

This work has been published previously in abstract form (Kisley and Gerstein, 1998).

\section{MATERIALS AND METHODS}

Female albino rats (Charles River, Wilmington, MA) weighing 250-350 gm were used for this study. Recordings were taken from both acutely prepared and chronically implanted animals. Results are presented as from a single population. The chronically implanted animals were also being used for a separate study involving recording during the performance of various auditory discrimination tasks. Detailed differences in procedures are noted below. All procedures are in accordance with the Institutional Animal Care and Use Committee at the University of Pennsylvania and the Society for Neuroscience's policy on the use of animals in neuroscience research.

Surgical procedures. Animals were initially anesthetized with an intraperitoneal injection of ketamine $(70 \mathrm{mg} / \mathrm{kg})$ and xylazine $(8 \mathrm{mg} / \mathrm{kg})$. Additionally, smaller doses were administered to maintain surgical anesthesia. Glycopyrrolate $(0.06 \mathrm{mg} / \mathrm{kg})$, a synthetic atropine antagonist, was injected subcutaneously to prevent respiratory tract secretions. Body temperature was monitored and maintained at $37^{\circ} \mathrm{C}$ with a fluid-filled heating pad.

Once surgical anesthesia was achieved (checked with pedal-withdrawal reflex), the head was shaved and fixed in a stereotaxic apparatus. Skin, muscle, and connective tissue were then cleared from the top and left temporal portion of the skull. Small stainless-steel screws were driven into holes drilled with a dental drill on the top of the skull. For acute experiments, the head of a long screw was affixed to the skull screws with dental acrylic. This "upward-facing" screw would later be attached, through a holder, to the operating table so that the stereotaxic ear bars could be removed. In this condition the head was held rigidly, but the ears were clear for auditory stimulation. A small craniotomy, $\sim 1 \mathrm{~mm}$ in diameter, was made with a dental drill over stereotaxic coordinates 5.0 $\mathrm{mm}$ posterior and $4.0 \mathrm{~mm}$ ventral of bregma, corresponding to left primary auditory cortex (Paxinos and Watson, 1997). After the dura was cut with a fine needle, microwire electrodes were rapidly inserted into the brain, then slowly ( $\sim 10 \mu \mathrm{m} / \mathrm{min})$ lowered to the infragranular layers $(0.8-1.2 \mathrm{~mm})$. Once at their desired depth, the electrodes were fixed in place using dental acrylic. Recordings for acute experiments were begun once the extracellular action potential waveforms appeared stable. For chronically implanted animals, dental acrylic was then liberally applied over the exposed skull and around a connector (Microtech, Boothwyn, PA), the skin on the head was sutured, and the animal was allowed to recover in isolation for 1 week. These animals were later reanesthetized with the same regimen to acquire data for the present study.

Electrophysiology and sensory stimulation. Both single wire electrodes and tetrodes were used in this study. Single wires were either stainless steel or gold-plated Ni-Ch, 50- $\mu \mathrm{m}$-diameter microwires (California Fine Wire, Grover Beach, CA). Tetrodes consisted of four $25 \mu \mathrm{m}$ tungsten microwires twisted together. Impedance at $1 \mathrm{kHz}$ for all wires was $<500 \mathrm{k} \Omega$.

Signals were buffered near the head with operational amplifier follower circuits, then passed through preamplifier, amplifier, and digital signal processing hardware (Plexon, Dallas, TX). Before the preamplifier, signal lines were split into two channels: extracellular action potentials ("units") and local field potentials. Unit data were filtered between 300 and $3000 \mathrm{~Hz}$, and candidate spike-waveforms were sampled at $40 \mathrm{kHz}$. These waveforms were stored for later spike sorting analysis. Field potentials were bandpass-filtered between 0.5 and $300 \mathrm{~Hz}$ and sampled continuously at $1 \mathrm{kHz}$. All signals were referenced to a ground wire securely attached to the skull screws.

Clicks ( $0.6 \mathrm{msec}$ square waves) were presented from a speaker located $22 \mathrm{~cm}$ from the right ear, $45^{\circ}$ to the right of the animal's horizontal body axis, $0^{\circ}$ azimuth. For some analyses, clicks were presented randomly with an interclick interval between 1.5 and $3.0 \mathrm{sec}$. Clicks were also presented in a manner time-locked to the ongoing local field potential activity as follows: for each single trial, one of several preselected delays was randomly selected. Once the ongoing field potential activity crossed an amplitude threshold, the delay was imposed and a click was presented. After $1.5 \mathrm{sec}$, another delay was randomly selected and so on. The timing of stimulus presentation was controlled with a Lab-PC board (National Instruments, Austin, TX) and accompanying software routines. All experiments were performed in a double-walled, sound-attenuated room.
Spike sorting and analysis. Bullock's (1997) definition of "multi-unit" activity was used in this report. All threshold crossings, regardless of waveform size or shape, were considered part of the multi-unit activity. Threshold levels were arbitrary but held fixed for an entire experiment. Generally, it appeared that between 5 and 10 single units contributed to the multi-unit recordings.

Often, several single units could be sorted out for individual analysis. For tetrode recordings, sorting was accomplished by making a scatter plot of the peak-to-peak amplitude of an action potential on one electrode versus the peak-to-peak amplitude of the same action potential recorded on another electrode of the tetrode (McNaughton et al., 1983; Gray et al., 1995). For single wires, sorting was accomplished by making a scatter plot of a waveform's projection onto the first principal component taken from a library of prerecorded waves versus the waveform's projection onto the second principal component (Abeles and Goldstein, 1977). For both sorting techniques, well isolated single units will tend to form well isolated clusters in the scatter plots.

Unit data were analyzed with peristimulus time histograms. The "centroid" of these histograms was computed as the mean latency of all spikes that occurred between 5 and $30 \mathrm{msec}$ from the onset of the click. Rhythmicity of ongoing activity was assessed by autocorrelogram.

Analysis of evoked local field potentials. Each single trial evoked waveform, $U_{\mathrm{i}}$, can be represented as a series of discrete values:

$$
U_{\mathrm{i}}=u_{\mathrm{ij}}\{\mathrm{j}=1,2, \ldots, m\},
$$

where time between samples is $1 \mathrm{msec}$, and $m$ is the number of points per waveform (either 100 or 128). In the first part of the study, the amplitude of single trial evoked responses was estimated using an "average-astemplate" method. The average wave is computed from all $n$ relevant waveforms using the standard method:

$$
\begin{aligned}
& \bar{U}=\bar{u}_{\mathrm{j}}\{\mathrm{j}=1,2, \ldots, m\} \\
& \bar{u}_{\mathrm{j}}=\frac{1}{n} \sum_{\mathrm{i}=1}^{n} u_{\mathrm{ij}},
\end{aligned}
$$

and then normalized (by the square-root of average power per point) to give the template waveform:

$$
\begin{aligned}
& \bar{V}=\bar{v}_{\mathrm{j}}\{\mathrm{j}=1,2, \ldots, m\} \\
& \bar{v}_{\mathrm{j}}=\frac{1}{b} \bar{u}_{\mathrm{j}} \quad b=\left[\frac{1}{m} \sum_{\mathrm{j}=1}^{m} \bar{u}_{\mathrm{j}}^{2}\right]^{1 / 2} .
\end{aligned}
$$

Single trial amplitude, $A_{\mathrm{i}}$, is the scalar result of taking the inner product of this normalized template and a single trial waveform:

$$
A_{\mathrm{i}}=\frac{1}{m} \sum_{\mathrm{j}=1}^{m}\left(u_{\mathrm{ij}} \cdot \overline{\mathrm{v}}_{\mathrm{j}}\right)
$$

Thus not only larger deflections from zero, but also greater similarity to the average waveform, will lead to a larger single trial amplitude. For comparison with a more traditional method, single trial amplitudes were also computed as the magnitude between temporally restricted minima and maxima (peak-to-peak method).

In the second part of this report, analysis of waveforms was performed using PCA. For this analysis, each single trial waveform was upsampled (using interpolation) by a factor of 5. After the mean (DC offset) was removed from each wave, the principal components were computed from the eigenvectors of the signal covariance matrix by the method described in Glaser and Ruchkin (1976). Respective eigenvalues describe the amount of data variance accounted for by each principal component.

By its very nature, PCA allows partial reconstruction of a waveform from those basis vectors that account for the greatest amount of variance present in the data. Because the first two components, $F_{1}$ and $F_{2}$, generally accounted for $>90 \%$ of the data variance in our study, all single trials were represented with only these components:

$$
F_{1}=f_{1 \mathrm{j}}\{\mathrm{j}=1,2, \ldots, m\} \quad F_{2}=f_{2 \mathrm{j}}\{\mathrm{j}=1,2, \ldots, m\}
$$


First and second "scores" were computed by projecting each single trial waveform onto the first and second principal components, respectively:

$$
S_{1 \mathrm{i}}=\frac{1}{m} \sum_{\mathrm{j}=1}^{m}\left(f_{1 \mathrm{j}} \cdot u_{\mathrm{ij}}\right) \quad S_{2 \mathrm{i}}=\frac{1}{m} \sum_{\mathrm{j}=1}^{m}\left(f_{2 \mathrm{j}} \cdot u_{\mathrm{ij}}\right) .
$$

Each score squared represents the signal power accounted for by that component. The sum-of-squares of all component scores is equivalent to the total power of the waveform, whereas the sum-of-squares of only the first two scores represents the power of the single trial waveform that can be fit by the shape of the first two components:

$$
P_{\mathrm{i}}=S_{1 \mathrm{i}}^{2}+S_{2 \mathrm{i}}^{2} \text {. }
$$

This quantity was taken as an estimate of the signal power for each waveform (the impact of this estimation is considered in Results). Because the first and second components are shaped differently, a quantitative estimate of shape for purposes of comparison between single trials is the fraction of waveform power accounted for by only the first component $\left(S_{1 \mathrm{i}}{ }^{2} / P_{\mathrm{i}}\right)$.

All data analysis was performed with custom-written programs and Matlab analysis package (Math Works, Natick, MA). All statistical estimations and hypothesis tests were performed under the assumption of normal distributions.

\section{RESULTS}

\section{Definition of anesthesia depth}

One way to assess state-dependent modulation of both evoked responses and response variability is to record such responses under different depths of anesthesia. In the present study we define three depths of ketamine/xylazine anesthesia: deep, medium, and light. This classification is based on our empirical observations of these relatively stable, easily defined states. During an experiment, these states can be cyclically repeated by giving small (approximately one-fourth the amount used for induction), supplemental doses of ketamine/xylazine every 90 or so min. Although the time course of transition between these states varies from rat to rat, deep anesthesia is generally stable by 20 min after a supplemental dose of ketamine/xylazine, medium from $\sim 50$ to $80 \mathrm{~min}$, and light after $\sim 80 \mathrm{~min}$.

Definition of anesthesia depth is achieved by a constellation of experimental parameters, the most important and reliable being the ongoing multi-unit activity. During deep anesthesia, multiunit activity exhibits low spontaneous rates and infrequent bursts or very slow $(<1.2 \mathrm{~Hz})$ rhythmic bursting. Medium anesthesia is characterized by higher spontaneous rates (usually $\sim 50 \%$ higher than deep anesthesia) and very clear rhythmic bursting activity at $\sim 1.6 \mathrm{~Hz}$. Under light anesthesia, multi-unit activity exhibits the highest spontaneous rates (often $>100 \%$ higher than deep anesthesia) and usually tonic firing patterns, but very occasionally rhythmic bursting $(>2 \mathrm{~Hz})$. Breathing patterns are also correlated with anesthesia depth. Breathing is very regular and slow ( $\sim 50$ breaths per min) under deep anesthesia. In medium anesthesia, breathing is a little faster (50-80 per min) but still regular. Breaths are significantly faster (100-160 per min) under light anesthesia and quite irregular. The transition from medium to light anesthesia is also accompanied by the beginnings of spontaneous whisker movement and a pedal-withdrawal reflex. The spectral composition of ongoing field potential activity is also somewhat correlated with depth of anesthesia, but generally depended too strongly on cortical depth to be useful in our recordings. With regard to the classic definition of anesthesia depth, all anesthetic levels are within stage III, "surgical," anesthesia (Thurmon et al., 1996). We do not necessarily intend our definitions to align directly with past attempts to delineate deep, medium, and
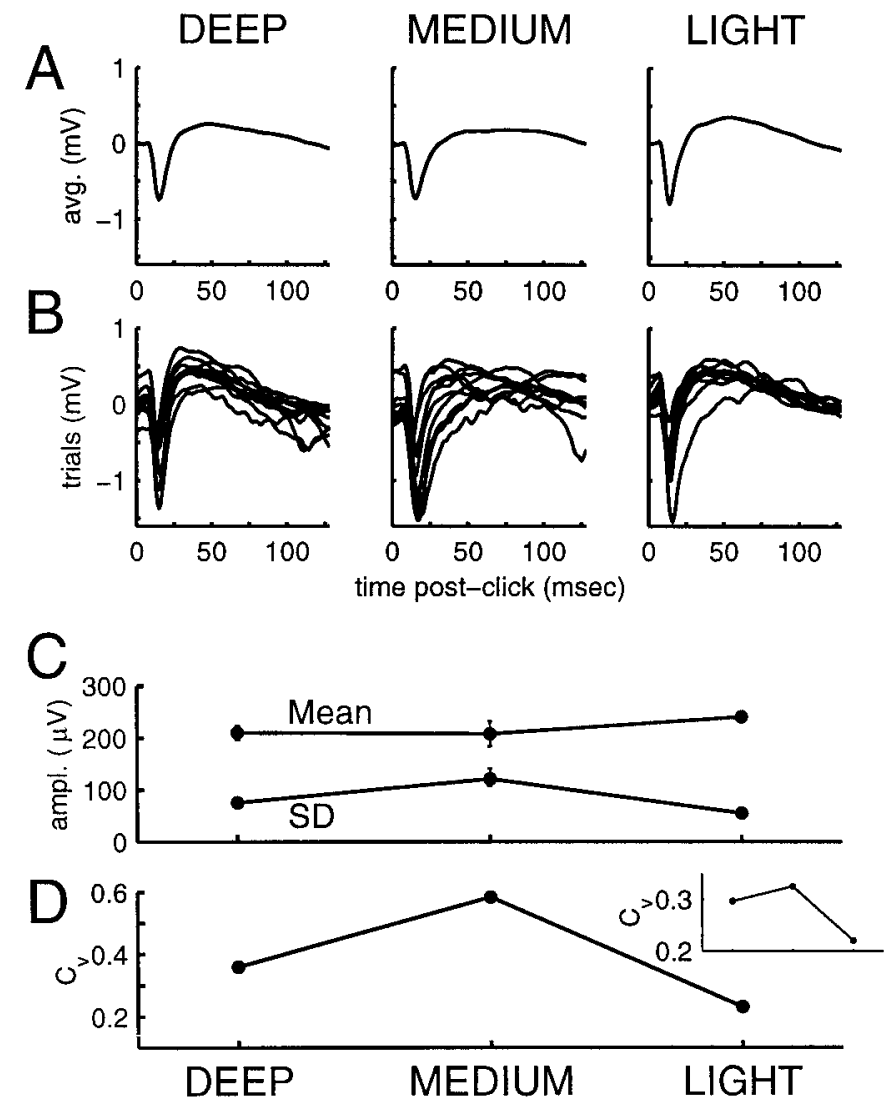

Figure 1. Depth of anesthesia (deep, medium, light) modulates clickevoked response and response variability. $A$, Average waveforms computed from 100-120 clicks at each anesthesia depth, presented randomly with interclick intervals between 1.5 and $3.0 \mathrm{sec}$. Click occurs at time $=0$ msec. Positive deflections of field potential are plotted upward. $B$, Ten example single trials for each state. Note the increased spread of waveforms at medium depth of anesthesia. $C$, Mean and SD of single trial amplitudes as estimated by taking the inner product with the normalized average waveform for each anesthesia depth. Error bars indicate $95 \%$ confidence intervals. $D$, Coefficient of variation $\left(C_{\mathrm{v}}=\mathrm{SD} /\right.$ mean $)$ as a function of anesthesia depth. Inset shows $C_{\mathrm{v}}$ as calculated using the traditional peak-to-peak method for estimating single trial amplitude. Although the general shape of the plot is similar, the coefficient of variation at the medium depth of anesthesia is substantially lower for this method.

light surgical anesthesia, which are based on many more physiological variables than we monitored.

\section{Modulation of response and response variability by anesthesia depth}

We found that average evoked responses differ slightly between the three anesthesia depths, but the single trial variability differs quite dramatically. This is shown for one rat in Figure 1. During this experiment, at least 100 click-evoked responses (random interstimulus intervals between 1.5 and $3.0 \mathrm{sec}$ ) were recorded under each anesthesia depth: deep, medium, and light. The general shape of these average evoked responses is quite similar to those recorded intracortically by Hall and Borbely (1970) in awake and sleeping rats. It can be seen from example single trials (Fig. $1 B$ ) that the evoked response is more consistent under deep and light, compared with medium, anesthesia. Not only does the peak amplitude vary widely under medium anesthesia, but even the overall time course (i.e., shape) varies significantly from one trial to the next. 


\begin{tabular}{lllll}
\hline \multicolumn{5}{c}{ Table 1. Size and variability of evoked responses for individual rats } \\
Rat & $\begin{array}{l}\text { Mean: } \\
\text { medium/light }\end{array}$ & $\begin{array}{l}\text { Variance: } \\
\text { medium/light }\end{array}$ & $\begin{array}{l}\text { Mean: } \\
\text { deep/light }\end{array}$ & $\begin{array}{l}\text { Variance: } \\
\text { deep/light }\end{array}$ \\
\hline A & $0.86^{a}$ & $2.60^{b}$ & 0.95 & $2.56^{b}$ \\
B & 1.17 & $1.96^{b}$ & & \\
C & & & 0.99 & 1.06 \\
D & 0.96 & $1.78^{b}$ & 0.93 & 1.34 \\
E & 0.88 & $4.17^{b}$ & $0.95^{a}$ & $2.16^{b}$
\end{tabular}

To allow direct comparison between rats, mean evoked response sizes and corresponding variances for medium and deep levels of anesthesia were normalized by equivalent variables under light anesthesia.

${ }^{a}$ Mean under medium or deep anesthesia is significantly less than mean under light anesthesia (large sample $z$ test, $\alpha=0.01$ ).

${ }^{b}$ Variance under medium or deep anesthesia is significantly more than variance under light anesthesia ( $F$ distribution test, $\alpha=0.01)$.

To quantify individual evoked field potential responses and their variability, estimates were made of each single trial amplitude by taking the inner product of each trial with a normalized average waveform (see Materials and Methods). This approach, rather than using just one or two points from each waveform, takes the shape and size of the entire wave into account. Statistics can then be estimated from the distribution of these single trial amplitudes (Fig. 1C, Table 1). Although evoked responses were generally largest under light anesthesia, only one of four rats exhibited significantly smaller evoked responses under medium and deep anesthesia (Table 1). On the other hand, the variance of evoked response was significantly higher under medium than under light anesthesia for all rats tested. In general, the variance increased from light to deep to medium anesthesia. This sensitivity of response variability to anesthesia depth can also be visualized with the coefficient of variation $\left(C_{\mathrm{v}}=\mathrm{SD} / \mathrm{mean}\right)$ (Fig. $1 D)$. Calculating single trial amplitudes with a peak-to-peak method yields basically similar results (inset). However, the coefficient of variation under medium anesthesia was quite a bit less than that computed using the inner product method. This confirms the notion that the peak-to-peak method is not very sensitive to variability of waveform shape.

\section{PCA of evoked responses under medium depth of anesthesia}

To characterize and even quantify the variability in waveform shape seen especially under medium depths of anesthesia, we used PCA. The goal of PCA is the same as Fourier analysis: to represent a waveform as a linear combination of a set of basis waveforms. In Fourier analysis the basis waveforms are predetermined (sine waves of different frequencies); in PCA the basis waves are computed from the data to be represented. In particular, the principal component bases explain the maximum amount of variance present in the data with the least number of waveforms. This aspect of PCA allows each waveform to be relatively well characterized by only a few parameters [for further discussion, see Glaser and Ruchkin (1976)].

Figure 2 shows the results of PCA on the 100 single trial evoked local field potentials recorded during a single session under medium anesthesia (the same set of waveforms shown in Fig. 1). For this set of data, the first component accounts for $63.7 \%$ of the variance of the data set, and the second for $27.0 \%$. Thus $>90 \%$ of the power in the population of waveforms can be described using the projection of each waveform onto only these two components. This percentage agrees with Musial et al. (1998) who recorded
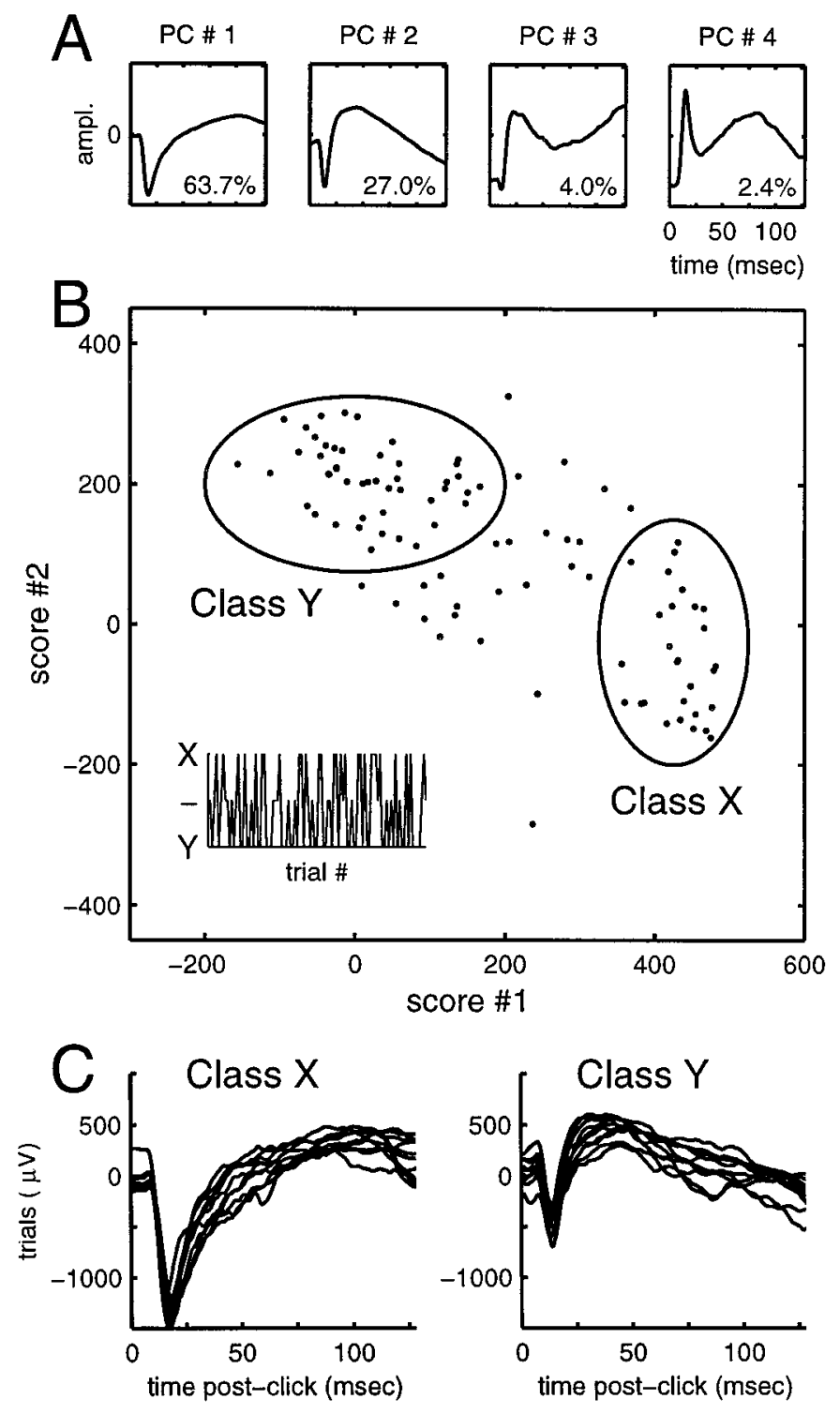

Figure 2. PCA allows arbitrary quantitative separation of single trials into response classes $(X$ and $Y)$. $A$, First four principal components for the medium depth of anesthesia data shown in Figure 1. The value in the bottom right of each plot corresponds to the percentage of total data variance accounted for by that component. $B$, Scatter plot of the first component score versus the second component score for each single trial. Selection of trials into either class $X$ or $Y$ is made with the ellipses. Inset shows chronological order of which single trials belong to Class $X$, Class $Y$, or neither $(-)$. $C$, Ten example single trials for each of the two classes. Note the striking difference between the two classes and the relative consistency within each class.

somatosensory evoked field potentials from barrel cortex of rats anesthetized with urethane.

The first and second component scores for each single trial waveform are the scalar results of taking the inner product of that waveform and the first and second principal components, respectively. A typical way of displaying component scores is a scatter plot, such as that shown in Figure $2 B$. Although not clearly distinct, there is some suggestion of two clusters of response types. We made these response types explicit by selecting responses as either class $\mathrm{X}$ or class $\mathrm{Y}$ with the illustrated ellipses. The inset of Figure $2 B$ shows that the variability of response 
shape, as summarized by class, was not caused by some slowly changing brain state, but rather a dynamic ongoing process.

Although the exact placement of the elliptical boundaries was somewhat arbitrary, Figure $2 C$ shows that classification succeeded in two ways. First of all, the general time course of the two response types differs quite substantially. Second, within each response class, the repeatability of the waveforms is relatively good. This latter point can be summarized quantitatively by comparing the average normalized inner product (i.e., correlation coefficient) between single trial waveforms before and after classification. The average normalized inner product between all 100 single trial waves is 0.43 . In contrast, this similarity measure is 0.94 for only the 28 class $\mathrm{X}$ waves, and 0.74 for only the 47 class Y waves. Therefore, PCA has allowed a numerically based separation into classes that reduced the observed time course variability of evoked field potential responses. It should be noted that the qualitative aspects of these results do not depend on the precise shape or location of the selection boundaries. In fact, separation into classes $\mathrm{X}$ and $\mathrm{Y}$-and the resulting reduction of variability — can be achieved nearly as well with a single straight line that roughly divides the two clusters of points (data not shown).

Evoked unit activity also shows quite dramatic differences between responses after PCA-based sorting of evoked field potentials. The click-evoked multi-unit activity, recorded simultaneously with the waveforms shown in Figure 2, is summarized in Figure 3. Figure $3 C$ is a peristimulus time histogram of the multi-unit activity for all waves. The time course of this multi-unit response generally matches the negative-going peak of the average evoked local field potential. This is expected because a negative deflection of the field potential represents negative extracellular currents, which corresponds to depolarization of nearby neurons. Figure $3 D$ shows the evoked multi-unit activity after PCA-based sorting into the two response classes. The later peak of the multi-unit activity for class X compared with class $\mathrm{Y}$ is correlated with the later peak in the evoked field potential waveform for class X. Also, the sustained unit discharge $(>30$ msec after the click) for class $\mathrm{X}$ responses could have been predicted from the sustained negative deflection of the class $\mathrm{X}$ field potential average. However, the difference between classes in spontaneous multi-unit activity directly preceding the clicks was quite unexpected. Note that there is virtually no spontaneous activity preceding a click when a class $\mathrm{X}$ evoked field potential occurs, compared with quite significant spontaneous activity preceding class $\mathrm{Y}$ evoked waveforms. This result suggests that the occurrence of a class $\mathrm{X}$ or a class $\mathrm{Y}$ evoked response depends on, and thus can be predicted from, the ongoing activity immediately preceding the click.

\section{Modulation of evoked response by ongoing activity}

To test the hypothesis that the observed variability in waveformshape was caused by, or at least correlated with, the ongoing cortical activity, we presented clicks at fixed delays from spontaneously occurring events. Figure 4 shows an average of 30 such events, which are very common during medium depths of anesthesia. From this figure it can be seen that a large negative spike in the field potential is accompanied by a large burst of multi-unit activity. In this particular recording, the burst events are occurring rhythmically at $\sim 1.6 \mathrm{~Hz}$ (Fig. $4 C$ ).

During an experiment, an amplitude threshold would be set on the ongoing field potential activity. When the threshold was crossed by a burst event, a fixed delay would be imposed and a
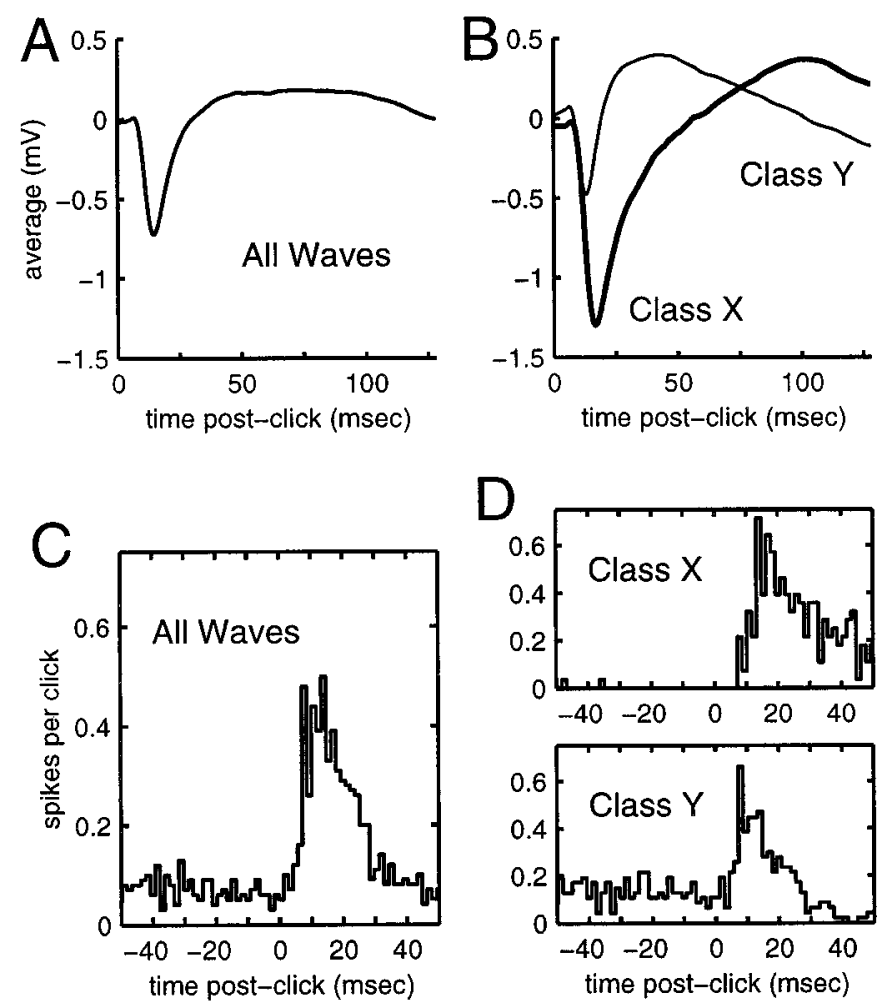

Figure 3. Evoked multi-unit activity differs for the response classes as defined by PCA. A, Average click-evoked field potential computed from all single trials for the data from medium depth of anesthesia in Figures 1 and 2. $B$, Average click-evoked field potentials for single trials as separated into Class $X$ (thick line) and Class $Y$ (thin line) by PCA. $C$, Multi-unit peristimulus time histogram for all single trials. Vertical axis shows the average number of spikes per click in each bin (bin size $=1.5$ $\mathrm{msec}$ ). Note that time scale is different than for field potentials. Click occurs at time $=0 \mathrm{msec}$. $D$, Multi-unit peristimulus time histograms after single trials have been sorted via PCA of corresponding field potential waveforms. Later peak latency and sustained negative deflection of class $\mathrm{X}$ field potentials correlates with later onset and sustained multi-unit discharges. Also note difference in spontaneous activity preceding clicks of the different response classes.

click presented. The delay was determined randomly for each single trial and could be either 150, 300, or $450 \mathrm{msec}$ (Fig. 4A). Responses were grouped together, or classified, on the basis of these delays.

We found that evoked responses are quite dramatically modulated by the spontaneously occurring population burst events in a manner that cannot be accounted for by linear superposition of a response and ongoing background activity. It can be seen from the single trials (Fig. 5A) that increasingly long delays from spontaneous threshold crossings lead to larger evoked responses and different time courses. There is no single evoked waveform that could be added to the average background waveform (Fig. $4 A$ ) at each of the different delays that would produce the observed time course distortions. Once again, these differences in waveform shape can be quantified with PCA. Figure $5 B$ shows the scatter plot of first and second component scores for all single trial evoked responses. Notice how the different symbols, corresponding to the different response classes, segregate quite well. Recall that these response classes are defined not by the analysis itself but rather by the relationship between each click and ongoing cortical activity.

Evoked multi-unit activity is also quite different depending on 


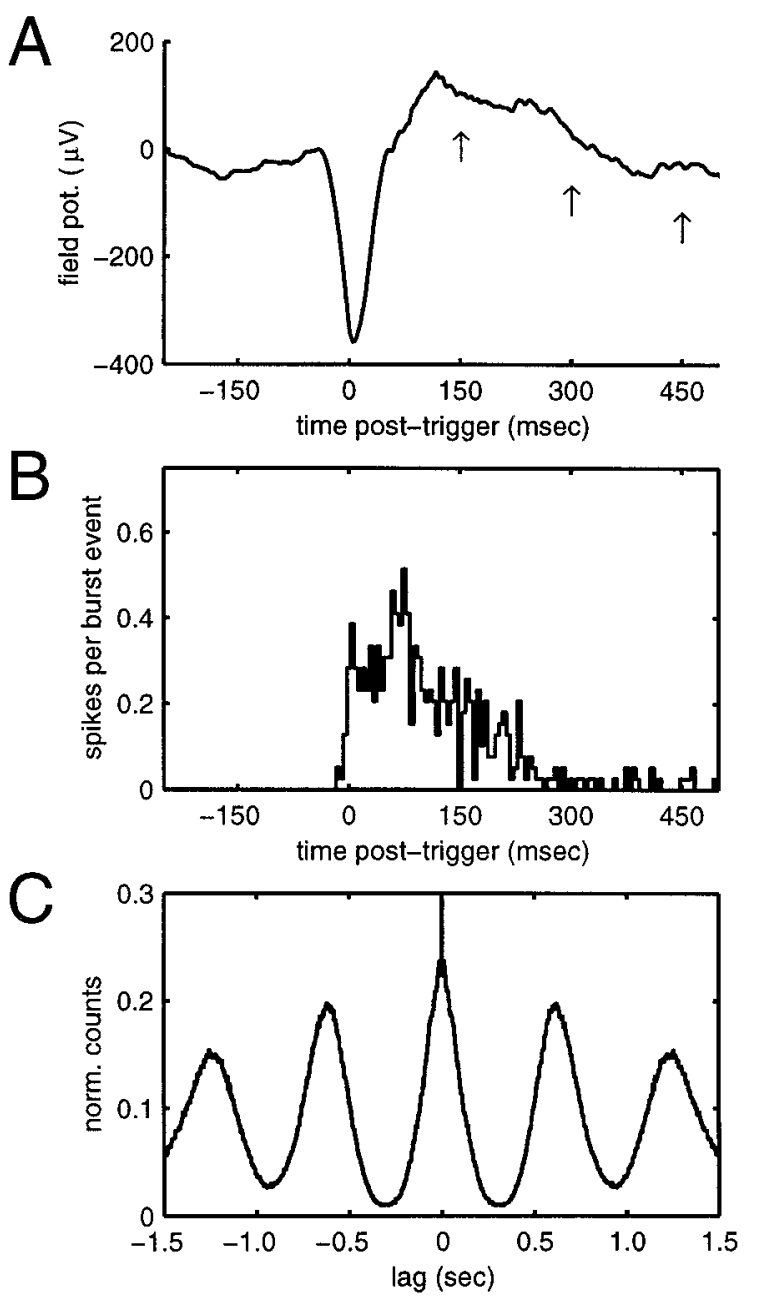

Figure 4. Population burst events occur spontaneously at medium depth of anesthesia. $A$, Average (field potential) of 30 spontaneous burst events, aligned on a threshold crossing $(0 \mathrm{msec})$. In subsequent figures, responses are sorted into classes that correspond to clicks presented at delays of 150 , 300 , and $450 \mathrm{msec}$ (indicated with arrows) from the burst event trigger crossing. $B$, Histogram of multi-unit activity locked to the same threshold crossing (bin size $=1.5 \mathrm{msec}$ ). $C$, Autocorrelogram of spontaneous multi-unit activity (bin size $=5 \mathrm{msec}$ ), normalized by height of central bin. Presence of secondary and tertiary peaks indicates rhythmic activity. Secondary peak occurs at a lag of $\sim 625 \mathrm{msec}$, which corresponds to a frequency of $\sim 1.6 \mathrm{~Hz}$.

how much time elapses between a population burst event and a click. In particular, notice the spontaneous activity preceding the clicks delayed by $150 \mathrm{msec}$ and the relative lack before the other clicks. In addition, there is a tendency for multi-unit activity to be sustained for a longer period of time after the click when it is delayed longer from the burst event.

The modulation of evoked field potential and multi-unit responses can be summarized quantitatively. For example, Figure $6 \mathrm{~A}$ shows a monotonic and statistically significant (large sample $z$ test, $\alpha=0.01$ ) increase in mean power of evoked field potential response as a function of delay from the population burst event. Note that, as opposed to response amplitudes calculated above, response power is computed as the sum-of-squares of the first two principal component scores for each wave (computing response power as the sum-of-squares of all component scores, i.e., total waveform power, yields a nearly identical relationship between
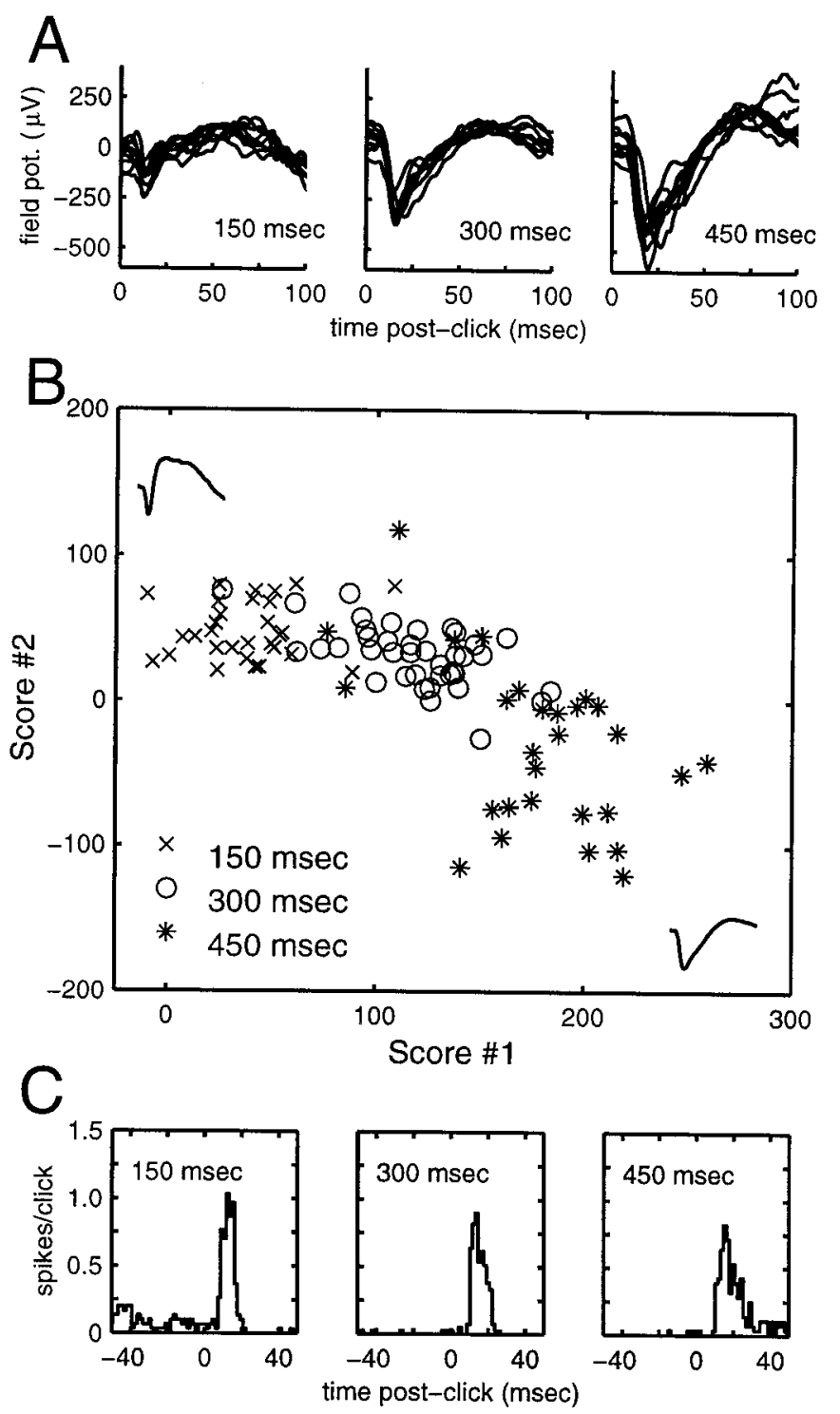

Figure 5. Spontaneous population burst events dynamically modulate evoked responses. $A$, Ten examples of single click-evoked field potentials (DC offsets removed) for the three different values of delay (indicated in bottom right of each plot) between spontaneous burst event and click. Note the change in both waveform size and shape with increasing delay. $B$, Scatter plot of first versus second principal component score for each single trial. Note how the different response classes (indicated by different symbols) segregate nicely in the principal component space. The shape of the first two components is shown near their respective axes. $C$, Multi-unit peristimulus time histograms for the three response classes (bin size $=1.5$ $\mathrm{msec}$ ). Increasing delay from the burst event leads to reduced spontaneous activity preceding the click, slower response onset, and longer response duration.

mean power and delay from burst event: correlation between the two methods of computing power is $>0.99$ in this instance). Differences in shape can be quantified by computing the percentage of a waveform's power accounted for by only one of the components, e.g., the first component. Plotting this value as a function of delay from a spontaneously occurring burst event (Fig. 6B) shows that longer delays (300 and $450 \mathrm{msec}$ ) lead to significantly modified evoked response shapes than a short delay $(150 \mathrm{msec})$. Figure $6 C$ indicates that mean latency of the negativegoing peak increases monotonically and significantly with increas- 


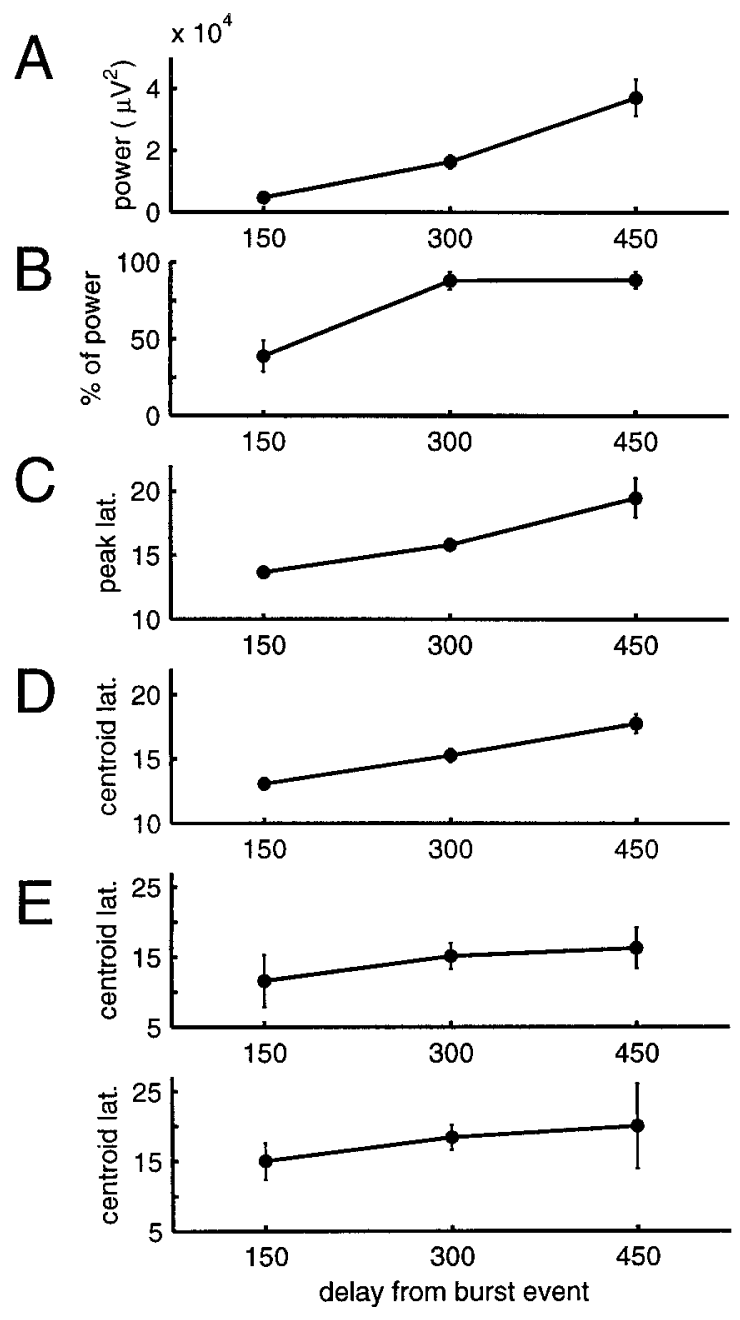

Figure 6. Summary of response modulation for data shown in Figure 5. $A$, Mean waveform power, estimated as the sum-of-squares of the first two principal component scores, as a function of click's delay from spontaneous burst event. $B$, Mean percentage of waveform power accounted for by the first principal component. $C$, Mean latency of peak (between 5 and 30 $\mathrm{msec}$ ) for single-trial evoked field potentials. $D$, Centroid latency (between 5 and $30 \mathrm{msec}$ ) for multi-unit histograms. $E$, Centroid latency for two single units recorded from different tetrodes $(\sim 300 \mu \mathrm{m}$ apart). Note change in vertical-axis scale. The trends for these units are the same as the multi-unit data, but there is large overlap of the confidence intervals because of relatively low spike counts. For all plots, error bars indicate $95 \%$ confidence intervals.

ing duration of delay from the spontaneous burst events, where the peak is the minimum value between 5 and 30 msec after a click. Related to this value, the centroid of the multi-unit peristimulus time histogram is computed by summing the latency of all spikes in the histogram between 5 and $30 \mathrm{msec}$ after the click, then dividing by the number of spikes. This temporal measure of multi-unit activity also increases steadily with larger delays from the burst events (Fig. 6D).

There are at least two ways to account for the change in latency of multi-unit response as a function of increasing delay from the population burst events. (1) The responses are composed of a combination of two separate neural populations that have different latencies of response (Wróbel et al., 1998). As delay from a burst event increases, the relative contribution of evoked activity to the multi-unit histogram shifts from being dominated by the earlier responding population to the later responding population. (2) Only one population of neurons constitutes the evoked multiunit response. As delay from a burst event increases, the actual latency of response of these neurons shifts. We believe the second explanation to be the most likely one in this particular situation because single units, isolated from the multi-unit population using tetrode-sorting techniques (see Materials and Methods), individually showed an increase in the centroid of their peristimulus time histograms as a function of delay from the burst events (Fig. $6 E$ ). This conclusion must be made cautiously, however, because the spike counts for single units were quite low, and some well isolated single units were not responsive to clicks. This is not surprising because auditory evoked single unit activity is somewhat suppressed under ketamine anesthesia (Zurita et al., 1994).

Although the spontaneously occurring population burst events were found to modulate click-evoked responses in all rats tested, the strength of modulation varied between rats and was usually but not always monotonically related to delay from the bursts. All five rats tested showed, with increasing delay from the burst events, increased mean latency of negative-going field potential peak (mean correlation coefficient $=0.78$ ), increased signal power (0.88), increased percentage of power accounted for by the first principal component (0.73), and increased latency of multiunit centroid ( 0.87 ; data only available for three rats). It should be noted that some rats were tested with different sets of delays. For example, in one rat, delays of 100, 200, 300, and $400 \mathrm{msec}$ from the burst events were used for the analysis. Using different delays in this manner did not yield qualitatively different results. Significantly longer delays could not be used because the burst events typically occurred rhythmically with mean inter-burst intervals of $\sim 600$ msec under medium depths of anesthesia.

Although not shown in the present report, we also found that responses to other types of auditory stimuli, e.g., tones and tone sweeps, are also dramatically modulated by these spontaneously occurring population burst events. Also, changing stimulus intensity does not particularly affect the character of the results. Finally, we found that burst events occurring during deep and light depths of anesthesia also modulate click-evoked responses. However, such burst events occur less frequently in deep and light anesthesia, and the degree of modulation is generally less than for similar events occurring during medium anesthesia. This observation explains why variability of evoked response was highest under medium levels of anesthesia.

\section{DISCUSSION}

We investigated trial-to-trial variability and state-dependent modulation of auditory cortex evoked responses in ketamine/ xylazine-anesthetized rats. We found that not only average responses but also response variability is modulated by the depth of anesthesia. In particular, it was shown that trial-to-trial variability is usually lowest under light anesthesia and highest under medium anesthesia. To quantify the observed variability in evoked waveform shapes, we used PCA. Such an analysis allowed classification of single trial evoked field potential responses into groups. This classification reduced single trial variability, and evoked multiunit activity was found to differ substantially among these groups.

We next examined whether the observed variability of waveform time course, as quantified with PCA, can be accounted for by the ongoing cortical activity. Indeed, by triggering the occurrence of auditory stimuli from the ongoing local field potential, it was found that large population burst events common to ketamine/xylazine anesthesia dynamically modulate both the size 
and shape of single trial evoked responses. In particular, under moderate depths of anesthesia, increasingly longer delays between the occurrence of a burst event and the presentation of a click lead to larger amplitude responses, modified response shapes, and later peak latencies. This modulation cannot be explained as a linear combination of ongoing activity and stereotyped evoked response.

\section{Potential cause(s) of observed variability}

The extreme variability of evoked responses observed under moderate levels of anesthesia could possibly be caused by the large-scale changes occurring throughout thalamocortical circuits during typical rhythms associated with sleep and anesthesia (for review, see McCormick and Bal, 1997; Steriade, 1997). For example, it is known that the responsiveness of both cortical and thalamic neurons varies with the phase of slow anesthesia rhythms (Contreras et al., 1996; Timofeev et al., 1996). The frequency of such rhythmicity in ketamine-anesthetized cats, typically $<1 \mathrm{~Hz}$ (Steriade et al., 1993), is lower than that which we observed (down to $\sim 1.2 \mathrm{~Hz}$ under deep anesthesia), but this discrepancy might be explained as a difference between species.

The presently observed rhythmic population bursting under medium levels of anesthesia more closely resembles spike-wave discharges, also known as high-voltage spindles (for review, see Coenen, 1995). These events, which can grow out of and likely share neural circuits with slow anesthesia rhythms (Steriade et al., 1998), are thought to be associated with certain forms of epilepsy (Snead, 1995). The basic phenomenology we observed roughly matches that shown by other researchers recording from rat cortex (Coenen, 1995; Kandel and Buzsáki, 1997; Seidenbecher et al., 1998). Although the frequency of spike-wave rhythmicity is typically $>2 \mathrm{~Hz}$, it has been previously observed down to $1.6 \mathrm{~Hz}$ in rats (Buzsáki, 1991) and $1.5 \mathrm{~Hz}$ in cats (Steriade et al., 1998). Ketamine, the anesthetic used in the present study, can induce spike-waves in cats (Black et al., 1980) and is often used to study neural activity during spike-waves (Steriade et al., 1998). Furthermore, ketamine has been shown to regulate the frequency of high-voltage spindle rhythmicity in rats (Buzsáki, 1991). Finally, it has previously been shown that presenting a visual stimulus in the interspike-wave interval causes a significantly different evoked potential than presenting a stimulus during the wave portion of a spike-wave, in a manner quite similar to our results [compare Fig. 5 of the present report with Fig. 5 of Pellegrini et al. (1986)].

Regardless of terminology, we believe the presently observed modulation of evoked responses to be primarily a reflection of oscillating excitability in both thalamic and cortical neurons. Immediately after a burst event there will be an accumulation of inhibitory currents that will cause a reduction in amplitude and even a modification in shape of evoked response. These might include $\mathrm{GABA}_{\mathrm{B}}$-mediated (Destexhe, 1998) and outward potassium currents (Steriade et al., 1998). After a sustained hyperpolarization, neurons would become primed to exhibit an enhanced (i.e., larger amplitude and extended time course) response to a sensory stimulus. This priming could be caused by deinactivation of voltage-gated sodium and perhaps also low-threshold calcium channels. These special calcium channels have been found to be involved in both thalamocortical rhythms and epilepsy [succinctly reviewed by Huguenard (1998); see de la Peña and GeijoBarrientos (1996) for cortical distribution of these channels]. It is also possible that the sustained inhibitory currents could activate hyperpolarization-activated cation currents (Pape, 1996) that would further prime the neurons.

\section{The linear model of ongoing background activity and evoked response}

One very critical issue to processing and interpreting neurophysiological data, and even to general cortical function, is whether relatively stereotyped evoked responses are linearly superimposed with ongoing brain activity. Such a condition is requisite for computing traditional average responses. Our results at first seem to contradict the optical recording study of Arieli et al. (1996) who found well behaved superposition of responses and background activity. However, the signals recorded are very different: local field potentials represent extracellular population currents (Bullock, 1997), whereas voltage-sensitive dye signals correspond to localized changes in neuronal membrane potentials (Arieli et al., 1995). More importantly, the stability of thalamocortical responsivity (i.e., excitability) apparently differed between our studies. Both Arieli et al. (1996) and Azouz and Gray (1999) indirectly assumed constancy of intrinsic responsivity and demonstrated sufficiency of the linear model under such circumstances. We have demonstrated conditions under which there is a systematic variation of responsivity and for which the linear model is deficient. The present results are probably most applicable to brain states associated with population bursting, rhythmic or not, such as natural sleep [Amzica and Steriade (1998); in humans: Achermann and Borbély (1997)], anesthesia, and paroxysmal episodes (see above).

\section{Variability and state-dependent modulation of evoked responses}

Our finding that variability of click-evoked response is lowest for light levels of anesthesia and highest under medium anesthesia is in direct contrast to Horvath (1969) who found that increasing depth of dial/urethane anesthesia leads to monotonically decreasing variability of surface-recorded auditory evoked potentials in cats. This discrepancy is most likely attributable to the use of different anesthetics, particularly because dial is a barbiturate. Barbiturates are known to cause generalized depression of central nervous activity (Thurmon et al., 1996) and have been specifically shown to reduce spontaneous activity in auditory cortex (Zurita et al., 1994). In contrast, as discussed above, moving from light to medium levels of ketamine/xylazine anesthesia increases the strength and prevalence of population burst events, each of which strongly modulates the shape and size of evoked responses, thus increasing the apparent variability of response.

Variability of long-latency $(>80 \mathrm{msec}$ ) auditory evoked responses has previously been investigated, especially in the human evoked potential field. For example, Zerlin and Davis (1967) showed that the peak-to-peak amplitude of the scalp-recorded N1-P2 complex (110-190 msec) is stochastic and obeys a Gaussian distribution. Especially pertinent to the present study, it has been shown that evoked potentials can be modulated by the phase of an ongoing rhythm during which they are presented [for review and alternative viewpoint see Rudell (1980)]. For example, Pfurtscheller (1976) found that an ongoing slow oscillation rhythmically modulated the amplitude of N90 waves recorded from awake subjects.

State-dependent modulation of shorter-latency evoked responses has previously been investigated in subhuman species. For example, the modulation of evoked potentials by different behavioral conditions (e.g., sleep and waking) has been examined in cat auditory (Herz, 1965), visual (Sigüenza et al., 1984), and somatosensory cortex (Howe and Sterman, 1973), and rat auditory (Hall and Borbely, 1970; Knight et al., 1985) and visual 
cortex (Bringmann and Klingberg, 1995) (for review, see Coenen, 1995). Also, modification of evoked potentials by spike-wave discharges has been studied in monkey (Mirsky et al., 1973), cat (Pellegrini et al., 1986), and rat visual cortex (Inoue et al., 1992; Meeren et al., 1998). In all of these studies only average waveforms were analyzed. By analyzing single trial waveforms we found that not only the average response but also response variability is modulated by brain state. Furthermore, in previous studies, average waveforms were computed from all evoked responses that occurred during a given behavioral state [see Pellegrini et al. (1986) for single exception]. We have shown that even during a fixed state, such as a particular depth of anesthesia, one evoked response can be significantly different than another because of very rapid transitions between $s u b$-states.

The finding that ongoing activity can modulate evoked responses in a ketamine/xylazine-anesthetized rat was not unexpected. Previously, Eggermont and Smith (1995) showed that ongoing activity, caused by a click, has a nonlinear impact on evoked field potentials generated by a subsequent click in ketamine/xylazine-anesthetized cats. Similarly, Kenmochi and Eggermont (1997) found that certain rhythmic click rates produce larger field potential deflections than others, suggesting again that activity evoked by preceding clicks can affect subsequent evoked responses. Furthermore, Barth and Di (1991) showed that directly stimulating rat somatosensory cortex leads to oscillatory changes between higher and lower levels of excitability, as measured with field potentials. One novel aspect of the present study is that the responses recorded were modulated by spontaneously occurring cortical activity, rather than by stimulation-evoked activity.

\section{REFERENCES}

Abeles M, Goldstein MH (1977) Multispike train analysis. Proc IEEE 65:762-773.

Achermann P, Borbély AA (1997) Low-frequency ( $<1 \mathrm{~Hz})$ oscillations in the human sleep electroencephalogram. Neuroscience 81:213-222.

Amzica F, Steriade M (1998) Cellular substrates and laminar profile of sleep K-complex. Neuroscience 82:671-686.

Arieli A, Shoham D, Hildesheim R, Grinvald A (1995) Coherent spatiotemporal patterns of ongoing activity revealed by real-time optical imaging coupled with single-unit recording in the cat visual cortex. J Neurophysiol 73:2072-2093.

Arieli A, Sterkin A, Grinvald A, Aertsen A (1996) Dynamics of ongoing activity: explanation of the large variability in evoked cortical responses. Science 273:1868-1871.

Astrelin AV, Sokolov MV, Behnisch T, Reymann KG, Voronin LL (1998) Principal component analysis of minimal excitatory postsynaptic potentials. J Neurosci Methods 79:169-186.

Aunon JI, McGillem CD, Childers DG (1981) Signal processing in evoked potential research: averaging and modeling. CRC Crit Rev Bioeng 5:323-367.

Azouz R, Gray CM (1999) Cellular mechanisms contributing to response variability of cortical neurons in vivo. J Neurosci 19:2209-2223.

Barth DS, Di S (1991) Laminar excitability cycles in neocortex. J Neurophysiol 65:891-898.

Black JA, Golden GT, Fariello RG (1980) Ketamine activation of experimental corticoreticular epilepsy. Neurology 30:315-318.

Bringmann A, Klingberg F (1995) Behavior-dependent and druginduced changes of rat visual evoked potential: relation to the EEG spectral power. Neuropsychobiology 31:89-97.

Bullock TH (1997) Signals and signs in the nervous system: the dynamic anatomy of electrical activity is probably information-rich. Proc Natl Acad Sci USA 94:1-6.

Buzsáki G (1991) The thalamic clock: emergent network properties. Neuroscience 41:351-364.

Chapman RM, McCrary JW (1995) EP component identification and measurement by principal components analysis. Brain Cogn 27:288-310.

Childers DG, Perry NW, Fischler IA, Boaz T, Arroyo AA (1987) Event- related potentials: a critical review of methods for single-trial detection. CRC Crit Rev Biomed Eng 14:185-200.

Coenen AML (1995) Neuronal activities underlying the electroencephalogram and evoked potentials of sleeping and waking: implications for information processing. Neurosci Biobehav Rev 19:447-463.

Contreras D, Timofeev I, Steriade M (1996) Mechanisms of long-lasting hyperpolarizations underlying slow sleep oscillations in cat corticothalamic networks. J Physiol (Lond) 494:251-264.

Coppola R, Tabor R, Buchsbaum MS (1978) Signal to noise ratio and response variability measurements in single trial evoked potentials. Electroencephalogr Clin Neurophysiol 44:214-222.

Dawson GD (1951) A summation technique for detecting small signals in a large irregular background. J Physiol (Lond) 115:2P-3P.

de la Peña E, Geijo-Barrientos E (1996) Laminar localization, morphology, and physiological properties of pyramidal neurons that have the low-threshold calcium current in the guinea-pig medial frontal cortex. J Neurosci 16:5301-5311.

Destexhe A (1998) Spike-and-wave oscillations based on the properties of $\mathrm{GABA}_{\mathrm{B}}$ receptors. J Neurosci 18:9099-9111.

Di S, Baumgartner C, Barth DS (1990) Laminar analysis of extracellular field potentials in rat vibrissa/barrel cortex. J Neurophysiol 63:832-840.

Eggermont JJ, Smith GM (1995) Synchrony between single-unit activity and local field potentials in relation to periodicity coding in primary auditory cortex. J Neurophysiol 73:227-245.

Gerstein GL (1960) Analysis of firing patterns in single neurons. Science 131:1811-1812.

Glaser EM, Ruchkin DS (1976) Principles of neurobiological signal analysis. New York: Academic.

Gray CM, Maldonado PE, Wilson M, McNaughton B (1995) Tetrodes markedly improve the reliability and yield of multiple single-unit isolation from multi-unit recordings in cat striate cortex. J Neurosci Methods 63:43-54.

Gur M, Beylin A, Snodderly DM (1997) Response variability of neurons in primary visual cortex (V1) of alert monkeys. J Neurosci 17:2914-2920.

Hall RD, Borbely AA (1970) Acoustically evoked potentials in the rat during sleep and waking. Exp Brain Res 11:93-110.

Heggelund P, Albus K (1978) Response variability and orientation discrimination of single cells in striate cortex of cat. Exp Brain Res 32:197-211

Herz A (1965) Cortical and subcortical auditory evoked potentials during wakefulness and sleep in the cat. Prog Brain Res 18:63-69.

Horvath RS (1969) Variability of cortical auditory evoked response. J Neurophysiol 32:1056-1063.

Howe RC, Sterman MB (1973) Somatosensory system evoked potentials during waking behavior and sleep in the cat. Electroencephalogr Clin Neurophysiol 34:605-618.

Huguenard JR (1998) Low-voltage-activated (T-type) calcium-channel genes identified. Trends Neurosci 11:451-452.

Inoue M, Van Luijtelaar ELJM, Vossen JMH, Coenen AML (1992) Visual evoked potentials during spontaneously occurring spike-wave discharges in rats. Electroencephalogr Clin Neurophysiol 84:172-179.

Kandel A, Buzsáki G (1997) Cellular-synaptic generation of sleep spindles, spike-and-wave discharges, and evoked thalamocortical responses in the neocortex of the rat. J Neurosci 17:6783-6797.

Kenmochi M, Eggermont JJ (1997) Autonomous cortical rhythms affect temporal modulation transfer functions. NeuroReport 8:1589-1593.

Kisley MA, Gerstein GL (1998) Variability of auditory cortex click responses as a function of preceding rhythmic activity. Soc Neurosci Abstr 24:1878.

Knight RT, Brailowsky S, Scabini D, Simpson GV (1985) Surface auditory evoked potentials in the unrestrained rat: component definition. Electroencephalogr Clin Neurophysiol 61:430-439.

Liberati D, Bertolini L, Colombo DC (1991) Parametric method for the detection of inter- and intrasweep variability in VEP processing. Med Biol Eng Comput 29:159-166.

McCormick DA, Bal T (1997) Sleep and arousal: thalamocortical mechanisms. Annu Rev Neurosci 20:185-215.

McNaughton BL, O'Keefe J, Barnes CA (1983) The stereotrode: a new technique for simultaneous isolation of several single units in the central nervous system from multiple unit records. J Neurosci Methods 8:391-397.

Meeren HKM, Van Luijtelaar ELJM, Coenen AML (1998) Cortical and thalamic visual evoked potentials during sleep-wake states and 
spike-wave discharges in the rat. Electroencephalogr Clin Neurophysiol 108:306-319.

Mirsky AF, Bloch S, Tecce JJ, Lessell S, Marcus E (1973) Visual evoked potentials during experimentally induced spike-wave activity in monkeys. Electroencephalogr Clin Neurophysiol 35:25-37.

Möcks J, Gasser T, Pham DT, Köhler W (1987) Trial-to-trial variability of single potentials: methodological concepts and results. Int J Neurosci $33: 25-32$.

Musial P, Kublik E, Wróbel A (1998) Spontaneous variability reveals principal components in cortical evoked potentials. NeuroReport 9:2627-2631.

Pape H-C (1996) Queer current and pacemaker: the hyperpolarizationactivated cation current in neurons. Annu Rev Physiol 58:299-327.

Paxinos G, Watson C (1997) The rat brain in stereotaxic coordinates, Ed 3. San Diego: Academic.

Pellegrini A, Zanotto L, Ermani M, Chemello R, Testa G (1986) Laminar field potentials and unit activity in the cortex during visual evoked potentials in feline generalized penicillin epilepsy. Exp Neurol 94:455-468.

Pfurtscheller G (1976) Variability of cortical evoked responses in man related to slow wave activity. Pflügers Arch 362:193-199.

Rose D (1979) An analysis of the variability of unit activity in the cat's visual cortex. Exp Brain Res 37:595-604.

Rudell A (1980) The rhythm of cortical excitability. Electroencephalogr Clin Neurophysiol 49:125-134.

Schiller PH, Finlay BL, Volman SF (1976) Short-term response variability of monkey striate neurons. Brain Res 105:347-349.

Scobey RP, Gabor AJ (1989) Orientation discrimination sensitivity of single units in cat primary visual cortex. Exp Brain Res 77:398-406.

Seidenbecher T, Staak R, Pape H-C (1998) Relations between cortical and thalamic cellular activities during absence seizures in rats. Eur J Neurosci 10:1103-1112.

Sigüenza JA, De Andres I, Ibarz JM, Reinoso-Suarez F (1984) Cortical visual evoked potentials during the sleep wakefulness cycle of the freely moving cat. Characterization and statistical comparisons. Electroencephalogr Clin Neurophysiol 59:165-171.

Snead OC (1995) Basic mechanisms of generalized absence seizures. Ann Neurol 37:146-157.
Softky WR, Koch C (1993) The highly irregular firing of cortical cells is inconsistent with temporal integration of random EPSPs. J Neurosci 13:334-350.

Steriade M (1997) Synchronized activities of couple oscillators in the cerebral cortex and thalamus at different levels of vigilance. Cereb Cortex 7:583-604.

Steriade M, Nuñez A, Amzica F (1993) A novel slow ( $<1 \mathrm{~Hz}$ ) oscillation of neocortical neurons in vivo: depolarizing and hyperpolarizing components. J Neurosci 13:3252-3265.

Steriade M, Amzica F, Neckelmann D, Timofeev I (1998) Spike-wave complexes and fast components of cortically generated seizures. II. Extra- and intracellular patterns. J Neurophysiol 80:1456-1479.

Thomas DG, Neer CM, Price JM (1989) Analyses of single-trial N1 amplitude and latency variability and their influence on the average evoked potential. Electroencephalogr Clin Neurophysiol 74:228-235.

Thurmon JC, Tranquilli WJ, Benson GJ (1996) Lumb and Jones' veterinary anesthesia, Ed 3. Baltimore: Williams \& Wilkins.

Timofeev I, Contreras D, Steriade M (1996) Synaptic responsiveness of cortical and thalamic neurones during various phases of slow sleep oscillation in cat. J Physiol (Lond) 494:265-278.

Tolhurst DJ, Movshon JA, Dean AF (1983) The statistical reliability of signals in single neurons in cat and monkey visual cortex. Vision Res 23:775-785.

Tunturi AR (1959) Statistical properties of near threshold responses to brief sounds in the MES auditory cortex of the anesthetized dog. Am J Physiol 196:1168-1174.

Vogels R, Spileers W, Orban GA (1989) The response variability of striate cortical neurons in the behaving monkey. Exp Brain Res 77:432-436.

Whitsel BL, Schreiner RC, Essick GK (1977) An analysis of variability in somatosensory cortical neuron discharge. J Neurophysiol 40:589-607.

Worden FG, Marsh JT, Abraham FD, Whittlesey JRB (1964) Variability of evoked auditory potentials and acoustic input control. Electroencephalogr Clin Neurophysiol 17:524-530.

Wróbel A, Kublik E, Musial P (1998) Gating of the sensory activity within barrel cortex of the awake rat. Exp Brain Res 123:117-123.

Zerlin S, Davis H (1967) The variability of single evoked vertex potentials in man. Electroencephalogr Clin Neurophysiol 23:468-472.

Zurita P, Villa AEP, de Ribaupierre Y, de Ribaupierre F, Rouiller EM (1994) Changes of single unit activity in the cat's auditory thalamus and cortex associated to different anesthetic conditions. Neurosci Res $19: 303-316$ 